\title{
CAMKs support development of acute myeloid leukemia
}

\author{
Xunlei Kang ${ }^{1,2^{*}+}$ (D), Changhao Cui ${ }^{1,4+}$, Chen Wang ${ }^{2,3}$, Guojin Wu' ${ }^{1}$, Heyu Chen ${ }^{1}$, Zhigang Lu' ${ }^{1}$ Xiaoli Chen ${ }^{1}$, Li Wang ${ }^{4}$, \\ Jie Huang ${ }^{5}$, Huimin Geng ${ }^{6}$, Meng Zhao ${ }^{3}$, Zhengshan Chen ${ }^{7}$, Markus Müschen ${ }^{7}$, Huan-You Wang ${ }^{8}$ \\ and Cheng Cheng Zhang ${ }^{1 *}$
}

\begin{abstract}
Background: We recently identified the human leukocyte immunoglobulin-like receptor B2 (LILRB2) and its mouse ortholog-paired Ig-like receptor (PirB) as receptors for several angiopoietin-like proteins (Angptls). We also demonstrated that PirB is important for the development of acute myeloid leukemia (AML), but exactly how an inhibitory receptor such as PirB can support cancer development is intriguing.

Results: Here, we showed that the activation of $\mathrm{Ca}(2+) /$ calmodulin-dependent protein kinases (CAMKs) is coupled with PirB signaling in AML cells. High expression of CAMKs is associated with a poor overall survival probability in patients with AML. Knockdown of CAMKI or CAMKIV decreased human acute leukemia development in vitro and in vivo. Mouse AML cells that are defective in PirB signaling had decreased activation of CAMKs, and the forced expression of CAMK partially rescued the PirB-defective phenotype in the MLL-AF9 AML mouse model. The inhibition of CAMK kinase activity or deletion of CAMKIV significantly slowed AML development and decreased the AML stem cell activity. We also found that CAMKIV acts through the phosphorylation of one of its well-known target (CREB) in AML cells.
\end{abstract}

Conclusion: CAMKs are essential for the growth of human and mouse AML. The inhibition of CAMK signaling may become an effective strategy for treating leukemia.

Keywords: Acute myeloid leukemia, CAMK, PirB, LILRB2, CREB, Leukemic stem cell

\section{Background}

Recently, we identified the human leukocyte immunoglobulin-like receptor B2 (LILRB2) and its mouse ortholog-paired Ig-like receptor (PirB) as receptors for several angiopoietin-like proteins (Angptls) [1]. LILRB2 and PirB are expressed by hematopoietic stem cells (HSCs) and leukemia stem cells in humans and mice, respectively, and support the ex vivo expansion of HSCs and acute myeloid leukemia (AML) development $[1,2]$. The level of LILRB2 mRNA is significantly higher in human acute monoblastic and monocytic leukemia cells (M5 subtype of AML) than in other AML cells [1]. A deficiency of PirB in the MLL-AF9 and AML1-ETO9a

\footnotetext{
* Correspondence: kangxu@health.missouri.edu;

Alec.Zhang@UTSouthwestern.edu

${ }^{\dagger}$ Equal contributors

${ }^{1}$ Departments of Physiology and Developmental Biology, University of Texas Southwestern Medical Center, 5323 Harry Hines Boulevard, Dallas, TX 75390, USA

Full list of author information is available at the end of the article
}

AML mouse models resulted in increased differentiation and decreased self-renewal of leukemia stem cells and significantly downregulated expression of a large number of tumor-promoting genes [1]. Although it was known that PirB inhibits the differentiation of myeloid-derived suppressive cells to stimulate solid cancer development [3], our results indicate that certain immune inhibitory receptors can be expressed on cancer stem cells and play essential roles in inhibiting differentiation and supporting self-renewal of these cells.

LILRB2 contains four extracellular Ig-like domains and a cytoplasmic portion with three immunoreceptor tyrosine-based switch motifs (ITIMs) [4]. The mouse ortholog PirB has six extracellular Ig-like domains and four ITIMs in its intracellular domain. The LILRB2/PirB ITIMs recruit tyrosine phosphatases SHP-1 and SHP-2 [5-7]. We were intrigued as to how inhibitory receptors such as LILRB2 and PirB support stem cell activity and cancer development. We found that binding of Angptls 
to PirB/LILRB2 induces activation of tyrosine phosphatase SHP-1/SHP-2 and calcium/calmodulin-dependent protein kinase CAMKIV [1], both known to be critical for HSC repopulation and stimulation of leukemia development $[1,2,8-10]$. We also found that SHP can induce CAMK activation in AML cells $[1,11]$.

CAMKs are serine/threonine kinases that are activated by increases of intracellular calcium concentration and that mediate subsequent cellular activities. Few studies have shown that CAMKs play important roles in hematopoietic physiology and pathology. CAMKIV is required for HSC activity; camk4-knockout mice have defects in HSC survival and proliferation [10]. CAMKII suppresses differentiation and stimulates proliferation of myeloid leukemia cells [9]. CREB is one of the well-known targets of CAMKs in hematopoietic cells, which can be phosphorylated and activated by CAMKs. This signaling plays a critical role in both normal hematopoiesis and leukemogenesis [11-13].

In this study, we sought to investigate the function of CAMKs in AML cells and understand how CAMKs support PirB signaling in AML development. We found that the expression of several CAMKs inversely correlates with the overall survival of human AML patients. Knockdown of CAMKs decreased human myeloid leukemia development in vitro and in vivo. In the mouse MLL-AF9 primary AML cells, the defective PirB signaling decreased CAMK activation, and the forced expression of CAMKs partially rescued the PirB-defective phenotype. Importantly, the inhibition of CAMK kinase activity or deletion of CAMKIV significantly lowered the activity of AML stem cells. By rescue assay, we also identified the phosphorylation of CREB is critical downstream for CAMKIV signaling in AML cells. Our results indicate that the CAMK signaling supports self-renewal and inhibits apoptosis of AML cells.

\section{Methods \\ Mice}

C57 BL/6 and NOD/SCID-IL2RG (NSG) mice were purchased from the UT Southwestern Medical Center animal breeding core facility or Jackson Labs. The PirB TM mice [14] and CaMKIV-null mice [10] were purchased from MMRRC and Jackson Labs, respectively. Mice were maintained at the UT Southwestern Medical Center animal facility. All animal experiments were performed with the approval of UT Southwestern Committee on Animal Care and University of Missouri Committee on Animal Care.

\section{Antibodies, reagents, and PCR primers}

Antibodies for flow cytometry, anti-Kit-APC, anti-Mac1-APC, anti-Gr-1-PE, anti-CD3-APC, and anti-B220-PE, were purchased from BioLegend and used as described [1]. The manufacturers and catalog numbers for other antibodies and reagents are as follows: anti-PirB-PE, R\&D Systems, FAB2754P; pCAMKI, Santa Cruz, sc-28438;
anti-pCAMKII, Abcam, ab32678; anti-pCAMKIV, Santa Cruz Biotechnology, sc-28443-R; anti-CAMKI, Abcam, ab68234; anti-CAMKII, Cell Signaling, 4436; antiCAMKIV, Cell Signaling, 4032; anti-pCREB, Cell Signaling, 9198S; anti-CREB, Cell Signaling, 9197S; anti-actin, Sigma Aldrich, A2066; STO-609, Sigma Aldrich, S1318; KN93, Sigma Aldrich, K1385; The PCR primer sequences were as follows: hCAMKI forward: CGGAGGACA TTAG AGACA, reverse: CTCGTCATAGAAGGGAGG-3; hCAM KIV forward: GATGAAAGAGGCGATCAG, reverse: TAGGCCCTCCTCTAGTTC. PirB forward: GAG AAT CACCAGACACATGC, PirB reverse: CTGCCCTCATG TCTTAACTT, mCAMKIV forward: AAGCAGGCGG AAGACATTAGG, CAMKIV reverse: AGTTTCTGAG TCCTCTTGTCCT.

\section{Virus infection and leukemia cell transplantation}

For retrovirus production, human embryonic kidney $293 \mathrm{~T}$ cells were grown in DMEM with 10\% fetal bovine serum (FBS) and transfected by MSCV-MLL-AF9-IRESYFP, MSCV-CAMKIV-IRES-GFP, MSCV-CAMKI-IRESGFP, or MSCV-CREB-IRES-GFP encoding plasmids and pCL-ECO to produce retroviruses. The infection of Linage-negative cells with retrovirus was performed as described previously $[1,15,16]$. Briefly, we infected Lin $^{-}$ cells with retroviral supernatant in the presence of $8 \mu \mathrm{g} / \mathrm{mL}$ polybrene. After infection, these cells were incubated overnight in medium with $10 \% \mathrm{FBS}, 20 \mathrm{ng} / \mathrm{mL}$ SCF, $10 \mathrm{ng} / \mathrm{mL}$ IL-3, and $10 \mathrm{ng} / \mathrm{mL}$ IL-6. Infected cells $(300,000)$ were transplanted into lethally irradiated (1000 rad) WT mice by retro-orbital injection. For secondary transplantation, $\mathrm{YFP}^{+}$ bone marrow $(\mathrm{BM})$ cells from primary transplanted mice were sorted and transplanted into mice with 200,000 normal BM cells as competitors.

For lentivirus production, the lentiviral vector Pll3.7 was used to express shRNAs to target CAMKI (sense: TGCCAGAGAATCTGCTGTACTATTCAAGAGATAGTA CAGC AGATTCTCTGGCTTTTTTC; antisense: GAAAAAAGC CAGAGAATC TGCTGTACTATCTCTTGAATAGTACA GCAGATTCTCTGGCA) and CAMKIV (sense: TGATAT TACAGTGAGCGAGATGTTCAAGAGACATCTCGCTC ACTGTAATATCTTTTTTGGAAC; antisense: TCGAGT TCCAAAAAAGATATTACAGTGAGCGAGATGTCTC TTGAACATCTCGCTCACTGTAATATCA). The lentivirus plasmid, pSPAX2, and pMD2.G (4:3:1) were transfected using PolyJet ${ }^{\mathrm{Tw}}$ In Vitro DNA Transfection Reagent (SignaGen Laboratories) into 293T cells. The resulting virus supernatant was collected $48-72 \mathrm{~h}$ later.

For Crisper-Cas9 system, we used pCW-Cas9 (gift from Eric Lander \& David Sabatini (Addgene plasmid \# 50661) [17]) to make stable MV4-11-Cas9 cell line, and designed three gRNA targeting the CAMK1 (gRNA1-GCTAC GACTTCCGAGATGTTC; gRNA2-GGAGCTCTTTGA CCGTATTGG; gRNA3-GATCCCGGTGTACAATGCCC) 
and one scramble gRNA (GAACGACTAGTTAGGC GTGTA).

\section{Flow cytometry, immunohistochemistry, and cytospin assays}

We performed flow cytometry, immunohistochemistry, and cytospin as described previously $[1,15,16]$. For flow cytometry analysis of AML cells, peripheral blood or BM cells were stained with anti-Mac-1-APC, anti-Gr-1-PE, anti-CD3-APC, anti-B220-PE, or anti-Kit-PE monoclonal antibodies (BioLegend). For analysis of apoptosis, indicated AML cells were stained with PE-conjugated anti-annexin V and 7-AAD (BD Pharmingen) according to the manufacturer's instructions.

\section{Human AML mouse xenograft model}

Human leukemia cell lines were cultured in RPMI supplemented with $10 \% \mathrm{FBS}$ at $37{ }^{\circ} \mathrm{C}$ in $5 \% \mathrm{CO}_{2}$ and the normal level of $\mathrm{O}_{2}$. Human leukemia cell lines were from ATCC. Xenografts were performed essentially as we described $[11,18]$. Briefly, adult NSG mice (6-8 weeks old) were sub-lethally irradiated with 250 cGy total body irradiation prior to transplantation. ShRNA-infected cells were resuspended in $200 \mu \mathrm{l}$ PBS containing 1\% FBS at a final dose of $1 \times 10^{6}$ human $\mathrm{GFP}^{+}$viable cells per mouse for retroorbital injection. One to 4 months after transplantation, the peripheral blood $(\mathrm{PB}), \mathrm{BM}$, spleen, and liver were assessed for engraftment by flow cytometry.

\section{CFU assays}

Five hundred $\mathrm{YFP}^{+} \mathrm{Mac}-1^{+} \mathrm{Kit}^{+} \mathrm{BM}$ cells from AML mice were plated in colony-forming unit (CFU) medium (M3534, Stem Cell Technologies) for CFU-GM assays, according to the manufacturer's protocols $[1,18]$. After 7 days, 2000 cells from three dishes were isolated for secondary plating.

\section{Patient overall survival analysis}

Data were obtained from the TCGA AML database (https://tcga-data.nci.nih.gov/docs/publications/tcga/; accessed September 5,2013), and levels were normalized to GADPH expression. Patients were divided into two groups based on whether their expression was above or below the median level of a gene.

\section{Western blotting}

Cell lysates (100 $\mu \mathrm{g}$ samples) were separated by electrophoresis on a 4-12\% SDS-polyacrylamide gel (BioRad), and the proteins were transferred onto a nitrocellulose membrane. The membrane was probed with indicated primary antibody for 1 hour at room temperature and then incubated with horseradish peroxidase-conjugated secondary antibody, which was detected with the chemiluminescence SuperSignal kit (Pierce).

\section{Statistical analyses}

Data are expressed as mean \pm SEM. For all experiments except determination of survival, data were analyzed by Student's $t$ test and differences were considered statistically significant if $p<0.05$. The survival rates of the two groups were analyzed using a log-rank test, and differences were considered statistically significant if $p<0.05$.

\section{Results}

CAMK activities are regulated by PirB signaling in AML development

Because LILRBs are highly expressed by monocytic AML (M5) cells [1], we used a retroviral MLL-AF9 transplantation mouse M5 AML model [19, 20] to investigate the relationship between PirB signaling and CAMKs in AML development. PirBTM mice [14], in which four exons encoding the transmembrane domain and part of the intracellular domain of PirB are deleted, were used to provide PirB-defective cells. PirBTM and control wild-type (WT) cells infected with MSCV-MLL-AF9-IRES-YFP retrovirus were transplanted to establish AML mice. We sought to determine whether the CAMK family decreased expression/activities in the PirB-defective MLL-AF9 AML mouse model. Compared to WT controls, PirBTM cells from MLL-AF9 AML mice had significantly decreased phosphorylation of CAMKI, CAMKII, and CAMKIV (Sun et al. [22]) (Fig. 1a), suggesting that CAMK activities are regulated by the PirB signaling pathway.

To test whether CAMK activities are essential to AML cells, we treated primary mouse MLL-AF9 AML cells with the CAMKK inhibitor STO-609 and CAMK inhibitor KN93. Both inhibitors diminished colonies in WT AML cells instead of PirB-deficient AML cells (Fig. 1b, c). These data show that the kinase activity of CAMK regulated by PirB signaling is critical for the growth of AML cells.

\section{CAMK transduction rescues PirB defects in AML}

We used gain-of-function approaches to further determine the role of CAMKI and CAMKIV in PirB signaling in AML cells. We introduced retroviruses encoding CAMKI or CAMKIV into PirB-defective AML cells to study whether CAMKs could rescue PirB defects in AML development. Mice transplanted with MLL-AF9-transduced PirBTM cells developed AML more slowly and survived longer than those transplanted with PirBTM cells that overexpressed CAMKI or CAMKIV (Fig. 1d). The PirBTM leukemia cells that overexpress CAMK exhibited accelerated development as demonstrated by the twofold increase in leukemia cell infiltration in the peripheral blood. This was detected using flow cytometry of these mice, compared to mice transplanted with PirBTM cells that did not overexpress CAMK, at 28 days post-transplantation (Fig. 1e). The in vitro CFU assay also showed both CAMKI and CAMKIV can increase PirBTM AML cells' colony-forming ability (Fig. 1h). 
a

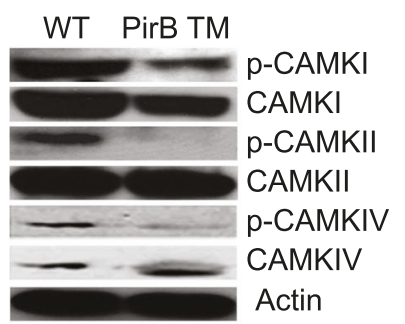

b

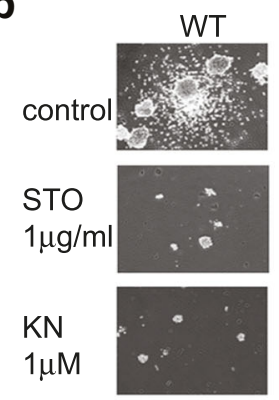

C

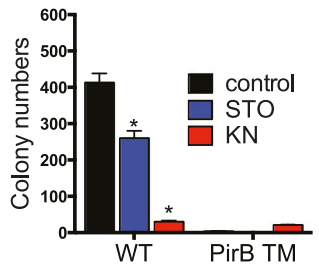

d

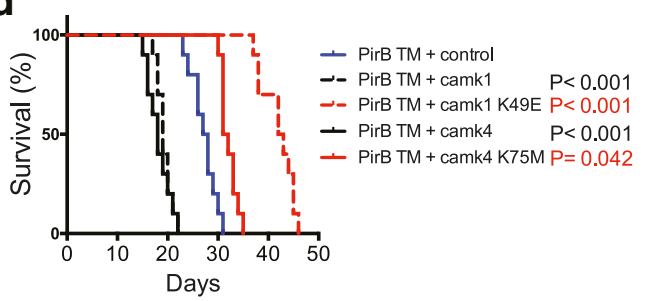

f

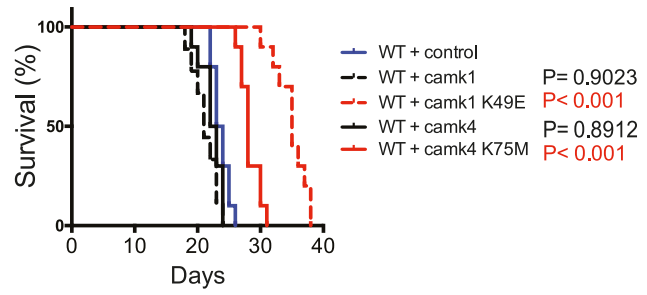

h

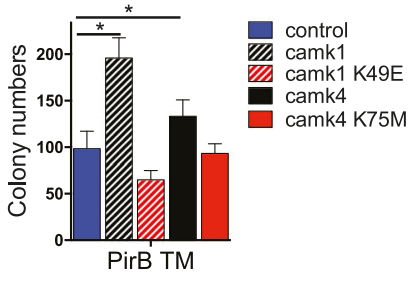

e

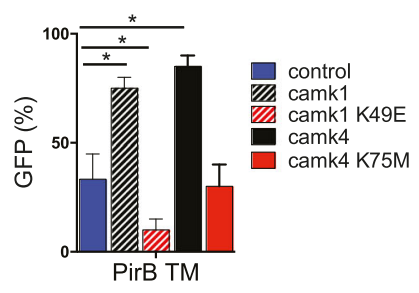

g

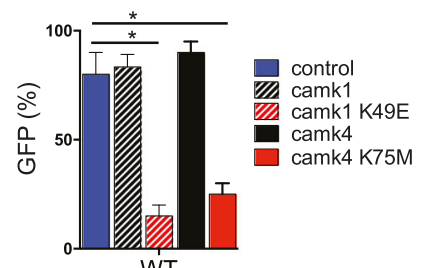

i

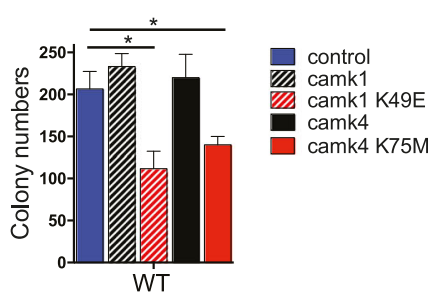

Fig. 1 Camk transduction enhances PirBTM MLL-AF9 AML development. a Phosphorylation of CAMKI, CAMKII, and CAMKIV was decreased in the PirBTM MLL-AF9 AML BM cells compared to WT cells. b, c Colonies formed from WT or PirB TM AML cells upon CAMK or CAMKK inhibitor treatment. Numbers of colonies formed by WT AML cells are decreased by addition of STO609 (STO) or KN93 (KN) ( $n=3)$. d, e, $\mathbf{h}$ Retrovirally expressed Camk1 and Camk4, but not Camk1 mutant (K49E) or Camk4 mutant (K75M), rescued PIRB TM phenotype upon secondary transplantation. Retrovirally expressed Camk 1 and Camk4 had similar levels as endogenous proteins in WT controls (Additional file 1: Figure S1). d Survival curves of mice transplanted with 3000 of these ectopically Camk1-expressing, Camk4-expressing, Camk1 K49E-expressing, Camk4 K75M-expressing, or control cells ( $n=15$ mice). e Percentages of retrovirus-infected (GFP+) AML cells in PB of secondary recipient mice after 28 days of transplantation. ( $n=5$ mice). $\mathbf{h}$ CFU numbers of retrovirus-infected $\left(\mathrm{GFP}^{+}\right) \mathrm{AML}$ cells in colony-forming assays. The experiment was repeated three times with similar results. $\mathbf{f}, \mathbf{g}$, i Retrovirally expressed Camk1 and Camk4 cannot change WT AML phenotype upon second transplantation. f Survival curves of mice transplanted with 3000 WT AML cells of these ectopically Camk1-expressing, Camk4-expressing, Camk1 K49E-expressing, Camk4 K75M-expressing, or control cells ( $n=15$ mice). g Percentages of retrovirus-infected $\left(G F P^{+}\right)$AML cells in PB of secondary recipient mice after 28 days of transplantation. ( $n=5$ mice). $\mathbf{i}$ CFU numbers of retrovirus-infected (GFP $)$ AML cells in colony-forming assays. The experiment was repeated three times with similar results; ${ }^{*} p<0.05$

Interestingly, in all of the rescue assays (Fig. 1d, e, h), neither the kinase-inactive CAMKI mutant (K49E) nor the CAMKIV mutant (K75M) was capable of supporting PirBTM leukemia development. We also performed the rescue assays in WT leukemia cells. Though CAMK overexpression did not affect WT leukemia development, the kinase-inactive CAMK1 mutant and CAMKIV mutant were able to play dormant negative roles in leukemia development in vivo and in vitro (Fig. 1f, g, i). These results demonstrate that CAMK, depending on their kinase activity, can rescue PirB defects in AML development, supporting our hypothesis that CAMKs are downstream mediators of PirB signaling. 


\section{CAMKIV supports mouse AML development during serial transplantation}

To gain a deeper understanding of the mechanism by which CAMKs support AML development, we sought to examine AML development in genetic CAMK deletion model. While CAMKI and CAMKII have multiple isoforms, CAMKIV exists as a single form. The availability of the Camk4-null mice [10] allowed us to focus on the role of CAMKIV as a representative of the CAMK family in the mouse AML model. It is clear that PirB and CAMKIV are highly expressed by hematopoietic progenitors and MLL-AF9 AML stem cell-enriched $\mathrm{Mac}-1^{+} \mathrm{Kit}^{+}$ population [11, 20, 21] (Additional file 1: Figure S2a-c).

The mice transplanted with MLL-AF9-transduced Camk4-null cells developed AML significantly more slowly than controls in both primary and secondary transplantation (Fig. 2a). The delayed development of the Camk4-null AML was also evident from the significantly decreased liver and spleen sizes and the numbers of white blood cells in circulation in the mice transplanted with Camk4-null cells compared to controls (Fig. 2b). The infiltration of Camk4-null myeloid leukemia cells into the liver and spleen was significantly decreased (Fig. 2c). There were more mature and differentiated hematopoietic cells especially the $\mathrm{Gr}-1^{+}$myeloid cells in mice that received Camk4-null cells than in those given control WT cells, based on cytospin and flow cytometry analyses (Fig. 2d-f). These results demonstrated that a lack of CAMKIV results in slower AML development.

We further analyzed whether CAMKIV affects AML stem cell (AML-SC) activity. Primary transplants with Camk4-null cells did not have significant differences in $\mathrm{YFP}^{+} \mathrm{Mac}^{+} \mathrm{Kit}^{+}$cells from mice transplanted with WT cells (Fig. 3a). In drastic contrast, the $\mathrm{YFP}^{+} \mathrm{Mac}^{+} \mathrm{Kit}^{+}$ cells in secondarily transplanted mice decreased from 53 to $30 \%$ in $\mathrm{BM}$ and from 11 to $1 \%$ in peripheral blood (Fig. 3b). A serial plating CFU assay was performed to test the self-renewal of AML-SCs in vitro. The deficiency of CAMKIV decreased CFUs in both primary plating and secondary plating, with dramatically lower numbers of colonies in secondary plating (Fig. 3c, d). Accordingly, CAMKIV was capable of rescuing the defects of PirBTM AML cells (Fig. 1h).

We also measured the apoptosis of Camk4-null and control AML BM cells. Overall, the MLL-AF9 BM AML cells had very low levels of early and late apoptosis (1.2 and $0.1 \%$, Fig. 3e). Levels of apoptosis were dramatically increased in the Camk4-null AML BM cells (4.6 and 0.9\%, Fig. 3e). These results suggest that CAMKIV supports the survival of AML cells. This is consistent with the report that CAMKIV is a critical survival factor for HSCs [10].

To quantitate how CAMKIV deficiency affects the AML-SC frequency, we performed transplantations by limiting dilution of sorted $\mathrm{YFP}^{+} \mathrm{WT}$ and Camk4-null
MLL-AF9 BM cells, collected from primary recipients. Leukemia development, as measured by survival ratio and latency days, is summarized in Fig. 3f. The frequency of AML-SCs was assessed and plotted as the percentages of non-leukemia-developing mice. Strikingly, the frequency of functional AML-SCs in Camk4-null primary MLL-AF9 AML model mice was only $1 / 12(=24 / 295)$ of that in control WT AML mice. This limiting dilution transplantation assay confirms that CAMKIV supports the activity of AML-SCs.

\section{CAMKs are essential for the growth of human leukemia cells}

Previously, we showed that the activation of LILRB2 induced CAMK activation. Similarly, based on our in silico analysis of human samples, the expression of LILRB2 and several CAMKs correlate inversely with the overall survival of AML patients (Fig. 4a-d), and CAMK1 is highly expressed by M5 AML cells (Fig. 4e) as LILRB2 [1]. These results suggest that CAMKs may play important roles in AML development. The activation of CAMKIV in human myeloid cells including the monoblastic KASUMI-1 cells and MLL-rearranged MV4-11 cells is greater than that in lymphoblastic leukemia cells such as KASUMI-2 and SUP-B15 cells (Additional file 1: Figure S3). The knockdown of CAMK1 or CAMK4 in AML cell lines including MV4-11 cells and KASUMI-1 led to significant inhibition of cell growth (Fig. $2 g-j$ ), indicating that CAMKs are functionally essential for human leukemia cells. By performing rescue assays (Fig. 4k), and Crisper assays (Additional file1: Figure S4) in MV4-11 cells, we proved the specific effect of CAMKI deficiency on AML cell growth.

Next, we tested the in vivo effect of knockdown of CAMK1 or CAMK4 expression in MV4-11 leukemia cells in transplanted NOD/SCID-IL2RG (NSG) mice. Both Camk1 or Camk4 knockdown significantly prolonged the survival of xenografted mice (Fig. 5a) and greatly inhibited leukemia development as determined by analysis of knockdown cells (Fig. 5b), human leukemic hCD $45^{+}$cells (Fig. 5c), and spleen size (Fig. 5d).

\section{CREB mediates the effect of CAMKIV on AML}

CREB was previously reported to be a possible downstream target of CAMKIV [22]. We found that PirBTM AML cells have decreased phosphorylation of both CAMKIV and CREB (Fig. 1a, Fig. 6a). Therefore, we hypothesized that CREB is a downstream mediator of PirB signaling in AML cells. To test this hypothesis, we introduced retrovirally expressed WT or inactive mutant CREB (S129A) into PirBTM AML cells. WT but not S129A CREB could rescue the defective phenotype of PirB TM AML cells in vitro and in vivo (Fig. $6 \mathrm{~b}-\mathrm{d}$ ). These results suggest that CREB acts downstream of PirB-mediated signaling to support AML development. 


\section{a}

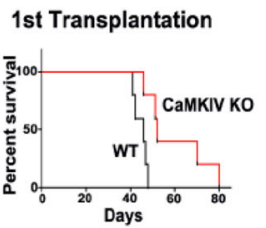

b

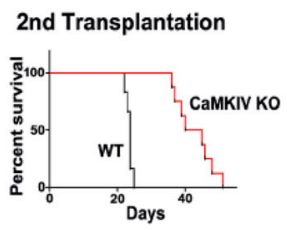

WT CaMkIV Ko WT CaMkIV Ko
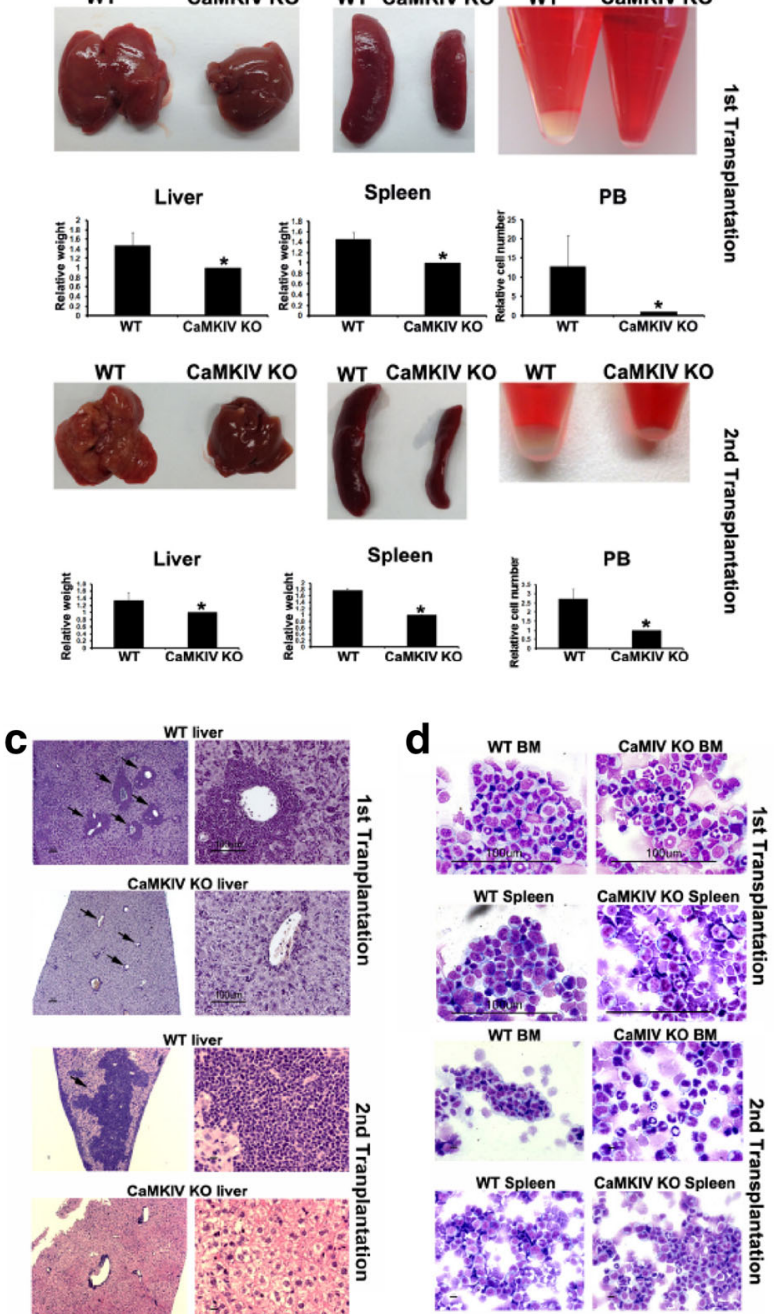
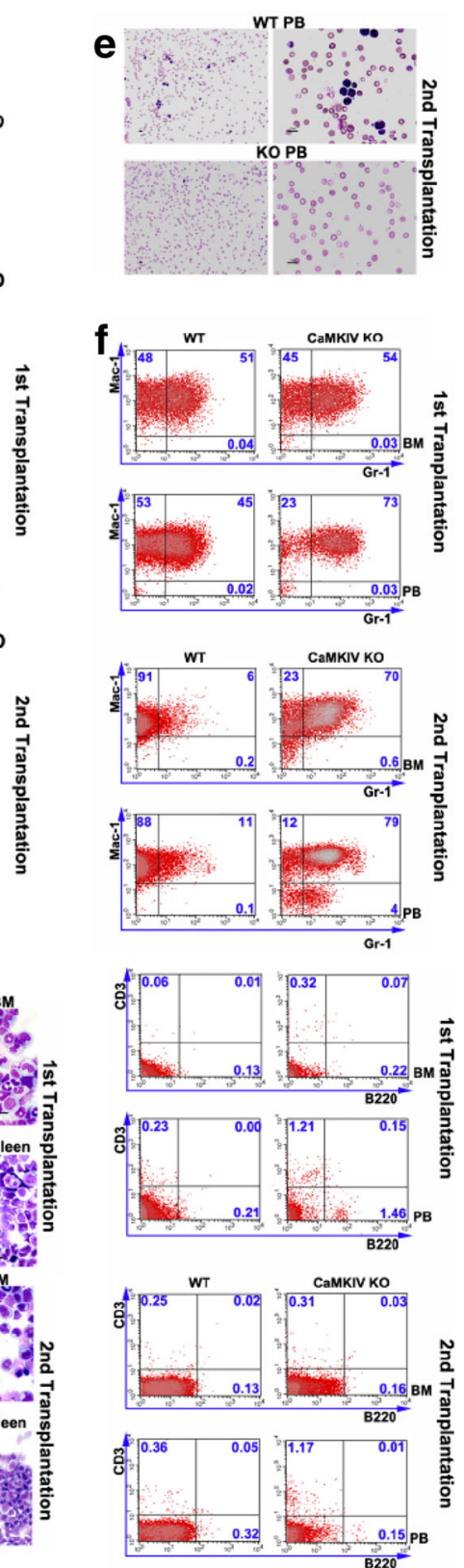

Fig. 2 Loss of CAMKIV decreases MLL-AF9 AML development. a Mice transplanted with MLL-AF9-infected WT hematopoietic progenitors had significantly reduced survival compared to mice transplanted with MLL-AF9-infected Camk4-null hematopoietic progenitors in both primary $(p=0.0019)$ and secondary $(p=0.0021)$ transplantation $(n=16)$. $\mathbf{b}$ Comparison of the sizes of spleens and livers and numbers of peripheral blood cells of the mice transplanted with WT MLL-AF9 cells and those with camk4-null MLL-AF9 cells at 41 days after first transplantation and 23 days after second transplantation. $\mathbf{c}$ Histological analysis of AML infiltration in the livers of mice transplanted with control or CAMKIV-transduced PirBTM AML cells (hematoxylin/eosin staining). Significant differences in AML infiltration to the liver in samples are indicated by arrows. $\mathbf{d}$, e Representative Wright-Giemsa-stained cytospin preparation of the BM, spleen, and peripheral blood cells from leukemic mice. $\mathbf{f}$ Representative flow cytometry plots show that camk4-null AML mice have more differentiated cells in BM and peripheral blood (PB) compared to mice transplanted with WT cells, at 23 days after transplantation. ${ }^{*} p<0.05$, scale bar is $100 \mu \mathrm{M}$

LILRB2 and CAMKs interact with each other in AML cells To test the relationship between LILRB2 and CAMKs, we transfected Flag-tagged LILRB2 and Myc-tagged CAMKI or CAMKIV into 293T cells, then performed co-immunoprecipitation and Western blotting assays. Both CAMKI and CAMKIV bind LILRB2 in transfected
293 T cells (Fig. 6e). Importantly, our bidirectional pulldown experiments showed that endogenous LILRB2 and CAMK1 interact with each other in MV4-11 cells (Fig. 6f). These data further support the conclusion that CAMKs play a crucial role in LILRB2-mediated signaling in AML cells (Fig. 6g). 
a

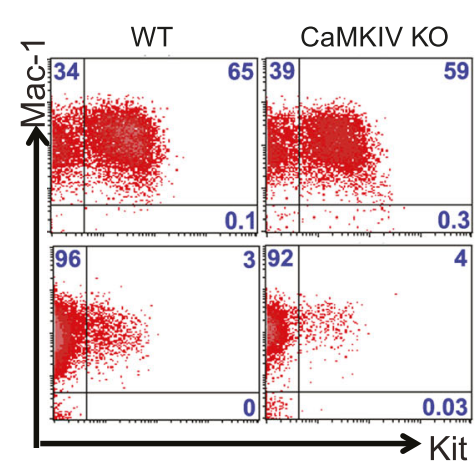

C

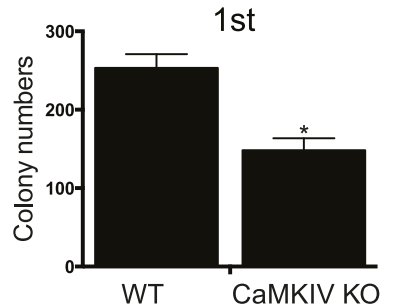

e

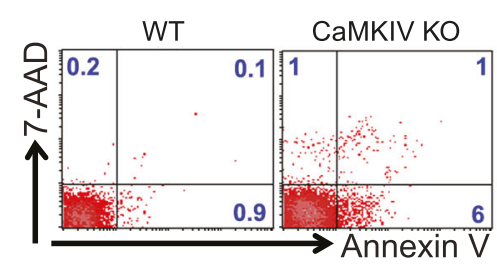

b
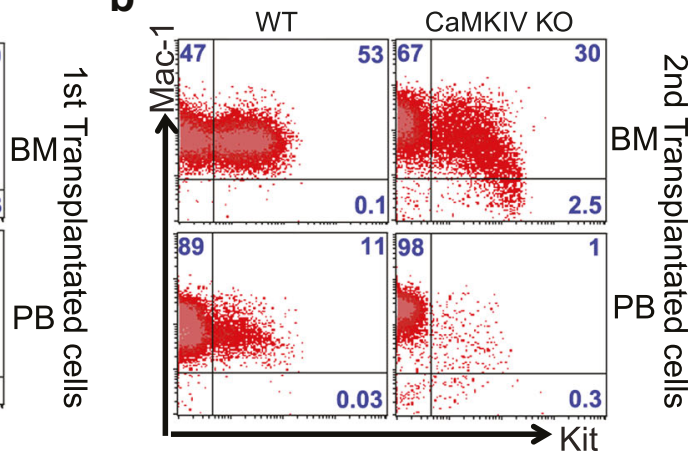

d
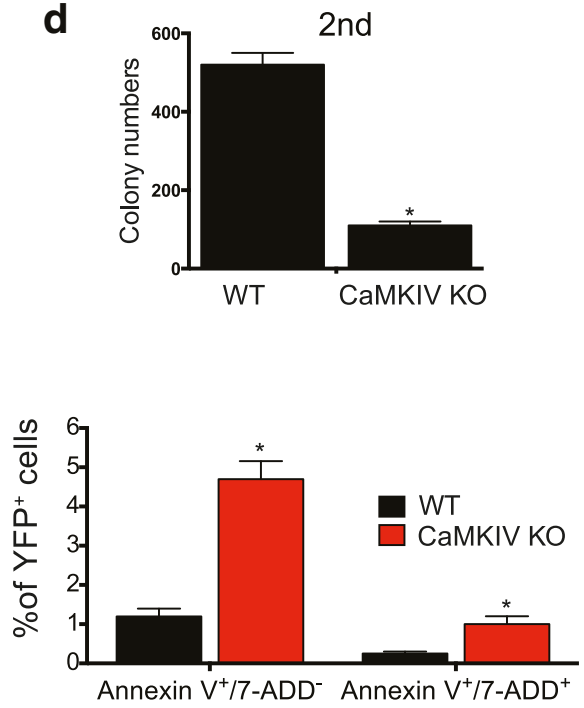

f

\begin{tabular}{|c|c|c|}
\hline Quantification of CRUs & \multicolumn{3}{|c|}{ survival ratio (latency days) } \\
\hline Transplanted cells & WT & CaMK IV KO \\
\hline 20 & $3 / 7(67.5)$ & $6 / 7(80)$ \\
\hline 200 & $0 / 7(47.7)$ & $4 / 7(68.9)$ \\
\hline 2000 & $0 / 7(22.4)$ & $0 / 7(42.8)$ \\
\hline 20000 & $0 / 7(16.6)$ & $0 / 7(19.8)$ \\
\hline $\begin{array}{c}\text { Frequency of tumor } \\
\text { initiating cells }\end{array}$ & $1: 24$ & $1: 295$ \\
\hline 95\% confidence interval & $(14-39)$ & $(181-482)$ \\
\hline
\end{tabular}

$\mathrm{P}<0.001$

Fig. 3 Loss of CAMKIV decreases self-renewal capacity of AML-SCS. a, b Representative flow cytometry plots show that Camk4-null MLL-AF9 AML mice had decreased numbers of $\mathrm{YFP}^{+} \mathrm{Mac}-1^{+} \mathrm{Kit}{ }^{+}$progenitors in both primary and secondary transplants. $\mathbf{c}$, $\mathbf{d}$ Primary and secondary transplants with Camk4-null MLL-AF9 BM cells show significantly decreased colony-forming ability $(n=3)$. e Camk4-null MLL-AF9 BM cells show increased

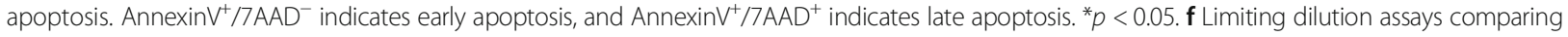
the frequencies of AML stem cells in WT and Camk4-null MLL-AF9 AML. The indicated YFP ${ }^{+}$WT and Camk4-null MLL-AF9 BM cells that were collected from primary recipients were co-transplanted with $2 \times 10^{5}$ bone marrow competitor cells into lethally irradiated recipients. The CRUs were calculated by L-Calc software

\section{Discussion}

Recently, we showed that Angptl binding to human LILRB2, or the mouse homolog PirB, stimulates phosphorylation of SHP-2 and CAMKIV [1, 2]. We sought to understand how immune inhibitory receptors support stem cell activity and cancer development. Here, we demonstrated that CAMKs play critical roles in sustaining AML-SC activity and PirB supports AML development at least partially through activation of CAMKs. To our knowledge, this is the first demonstration of how an ITIM-containing receptor signals, via activation of kinase activities, to support cancer development.

The positive roles of CAMKs in mouse and human leukemia development are supported by a variety of evidence as shown in our study. (1) The expression of CAMKs inversely correlates with the overall survival of 
a

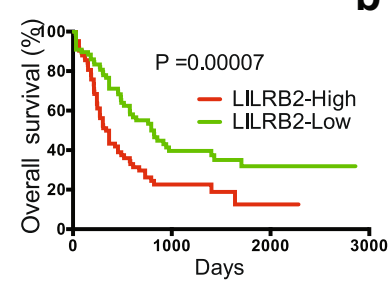

d

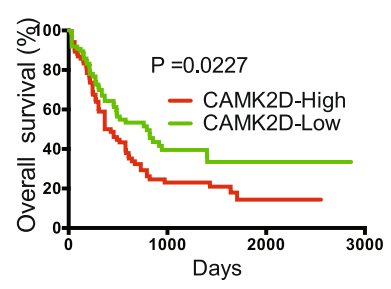

f

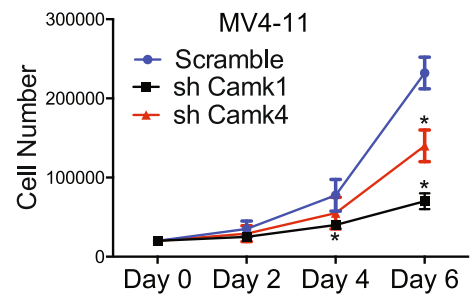

h

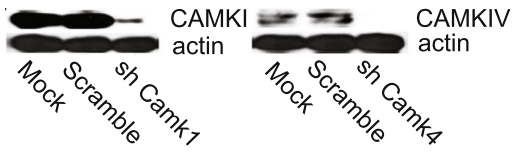

j

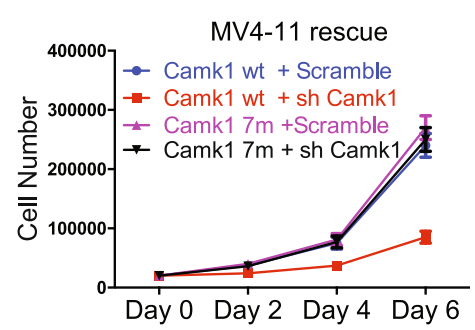

I

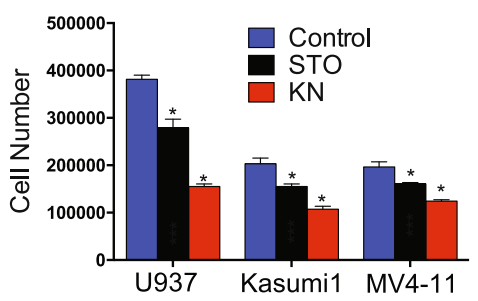

C

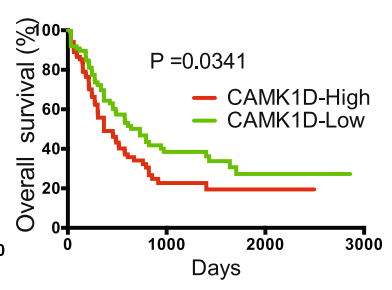

e

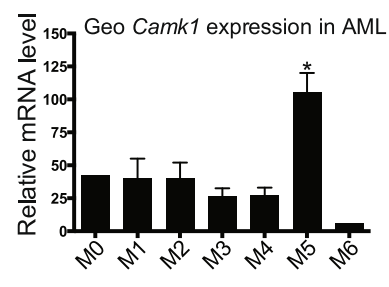

g

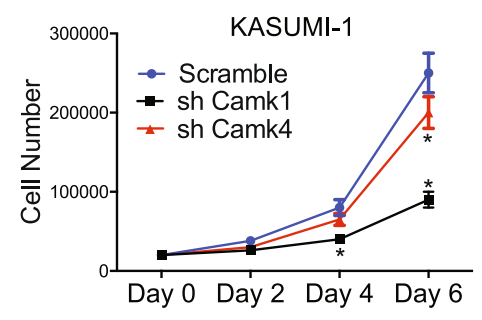

i

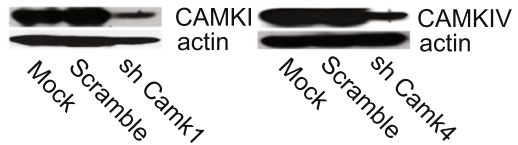

k

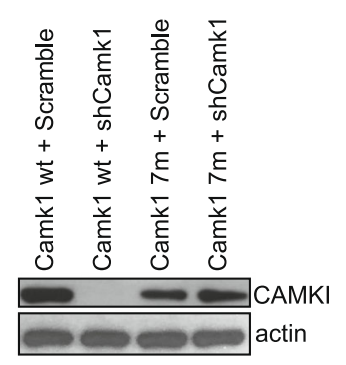

Fig. 4 (See legend on next page.) 
(See figure on previous page.)

Fig. 4 CAMKs play critical roles in human AML. a-d LILRB2 and CAMK expression negatively correlates with the overall survival of AML patients. The mRNA expression data of LILRB2 and CAMKs were obtained from the TCGA AML database (https://tcga-data.nci.nih.gov/docs/publications/ tcga/; accessed November 5, 2012) and normalized by GADPH expression. Patients were separated into two groups based on whether they have higher $(n=83)$ or lower $(n=82)$ than the average expression levels of the indicated genes $(n=186)$. e An in silico analysis of human Camk1 mRNA expression in 43 human AML samples as described previously [24]. f Treatment with shRNAs targeting Camk1 or Camk4 inhibited the growth of MV4-11 cells. GFP ${ }^{+}$cells were sorted by flow cytometry 1 day post-infection, and 20,000 cells were plated. Cell numbers were determined at three time points (days 2, 4, and 6) from triplicate wells. The experiment was repeated three times with similar results. $\mathbf{g}$ Inhibition of Camk1 or Camk4 expression inhibited the growth of KASUMI-1 cells. Cell numbers were determined at three time points (days 2, 4, and 6) from triplicate wells. The experiment was repeated three times with similar results. Knockdown of Camk1 and Camk4 in MV4-11 cells and KASUMI-1 cells as determined by Western blotting (h, i). $\mathbf{k}$ Rescue of Camk1-knockdown phenotype. Seven mutations were introduced into puromycin-seleted Camk1 expression lentivirus vector. Expression from this mRNA did not change CAMK1 amino acid sequence and was not silenced by shRNA Camk1. Mutant Camk1 (7 m) infected MV4-11 cells were resistant to the shRNA-Camk1-induced growth inhibition. The experiment was repeated three times with similar results. GFP ${ }^{+}$cells were sorted by flow cytometry 1 day post-infection, and 20,000 cells were plated. Cell numbers were determined on days 2, 4, and 6 in triplicate wells. The experiment was repeated three times with similar results. I Western blot showed mutant Camk1 could not be silenced by shRNA Camk1. m Cell growth of human AML cells (U937, Kasumi1 and MV4-11) were inhibited upon CAMK (STO609) or CAMKK inhibitor (KN93) treatment. Numbers of cells were calculated 6 days post treatment; ${ }^{*} p<0.05$

a

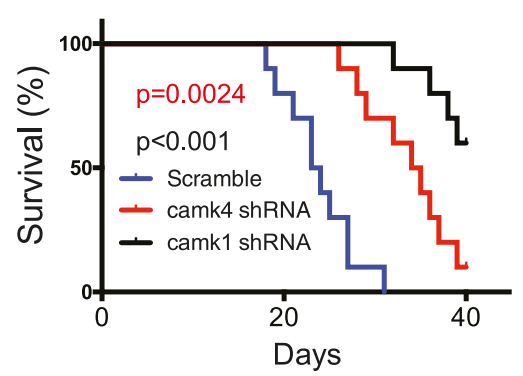

C

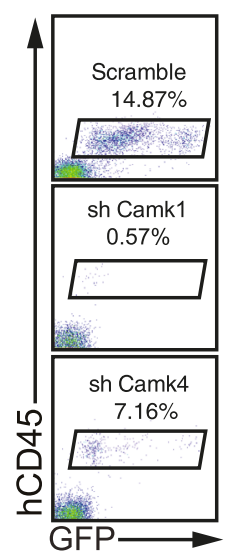

b

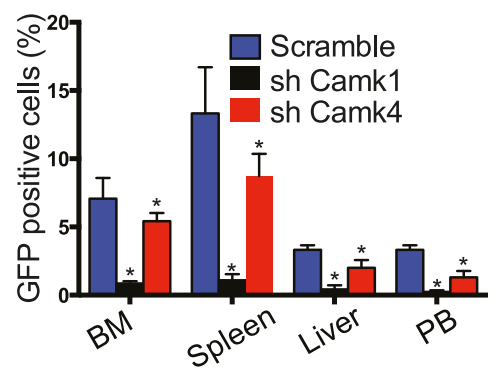

d

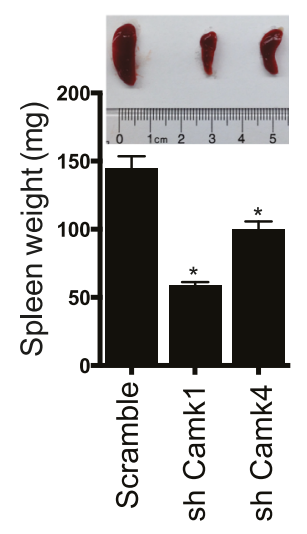

Fig. 5 Knockdown of CAMK1 or CAMK4 blocks xenograft of human leukemia cells. a Survival curve of NSG mice transplanted with MV4-11 cells $\left(1 \times 10^{6}\right.$ cells) infected with virus designed to express GFP and either scrambled shRNA, Camk1 shRNA, or Camk4 shRNA. GFP ${ }^{+}$cells were collected and transplanted into NSG mice 1 day post-infection ( $n=10$ mice). b Percentages of GFP' cells in BM, spleen, liver, and PB at 28 days after transplantation. $\mathbf{c}$ Representative flow cytometry plots indicating decreased spleen engraftment of MV4-11 cells treated with shRNA targeting Camk1 or Camk4. Staining with anti-human CD45 antibodies confirmed engraftment was from transplanted human leukemia cells. The percentages of hCD45 ${ }^{+}$population are indicated. $\mathbf{d}$ Comparison of the sizes of spleens of the mice transplanted with control MV4-11 leukemia cells or cells expressing shRNA targeting Camk1 or Camk4 ( $n=5$ mice); ${ }^{*} p<0.05$ 
a
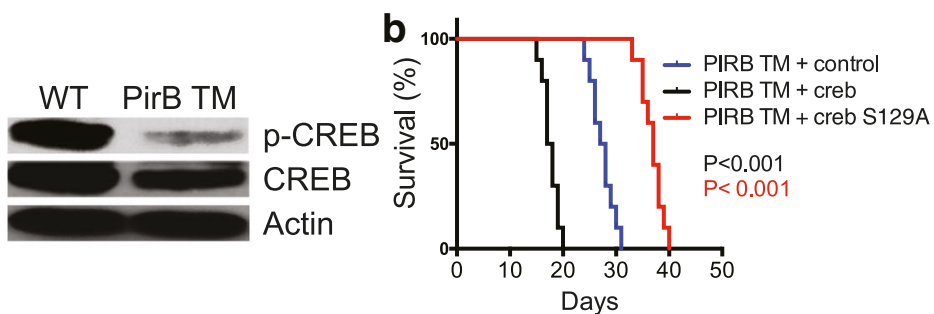

C

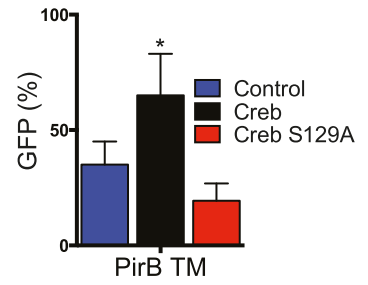

d

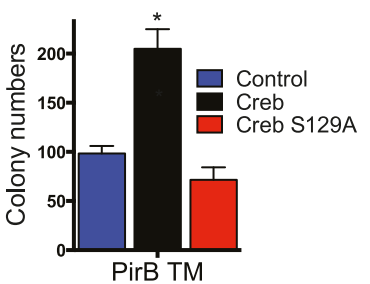

e
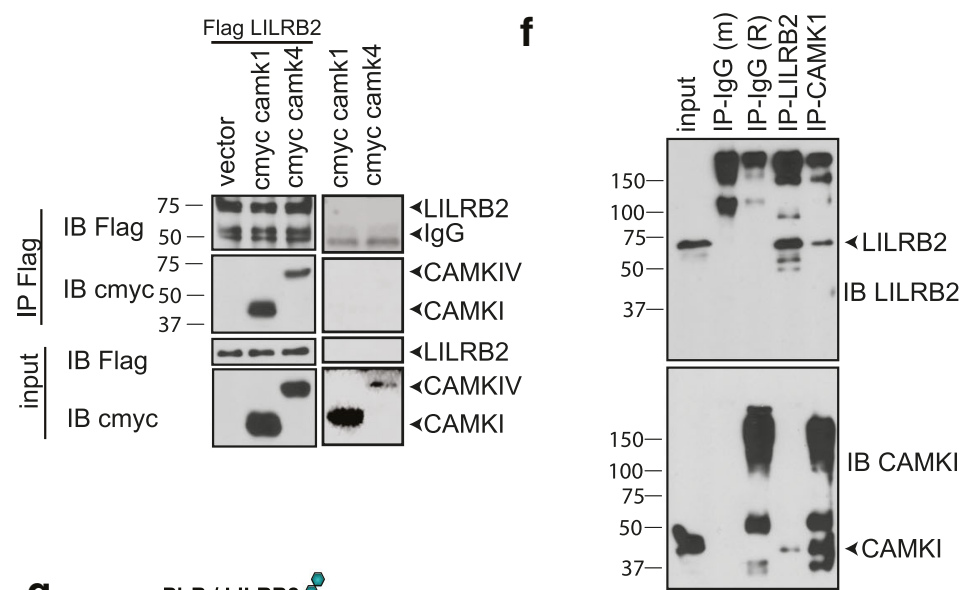

g

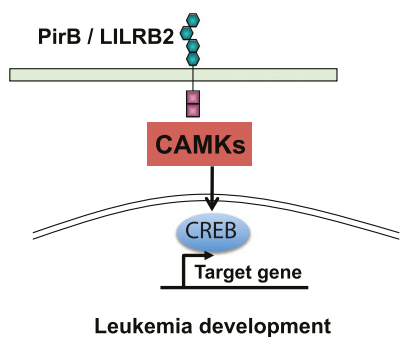

Fig. 6 CREB activity plays important role in PirB/LILRB2-CAMKs signaling in AML-SCs. a Phosphorylation of CREB was decreased in the PirBTM MLL-AF9 AML BM cells compared to control cells. b-d Retrovirally expressed Creb, but not Creb mutant (S129A), rescued PirBTM phenotype upon secondary transplantation. Retrovirally expressed Creb had similar levels as endogenous proteins in WT controls. b Survival curves of mice transplanted with 3000 of these ectopically Creb-expressing, Creb S129A-expressing, or control cells ( $n=10$ mice). c Percentages of retrovirus-infected (GFP ${ }^{+}$) AML cells in PB of secondary recipient mice after 28 days of transplantation. ( $n=5$ mice). $\mathbf{d}$ CFU numbers of retrovirus-infected (GFP $\left.{ }^{+}\right)$AML cells in colony-forming assays. The experiment was repeated three times with similar results; e CAMKI and LILRB2 bound in transfected 293T cells. The indicated flag-tagged LILRB2 or myc-tagged CAMKI or CAMKIV proteins were overexpressed in 293 cells. Flag antibody was used to precipitate LILRB2 proteins, and the flag or myc antibodies were used in Western blots. $\mathbf{f}$ Endogenous CAMKI and LILRB2 interact with each other as determined by bidirectional pull-down assays. MV4-11 cells $\left(1 \times 10^{7}\right.$ cells $)$ were lysed with transmembrane protein extraction reagent and indicated antibodies were used for immunoprecipitation and Western blot; ${ }^{*} p<0.05$. g Schematic summary of the signaling pathway mediated by the PirB/LILRB2-CAMKs-CREB in AML cells

AML patients. (2) Knockdown of CAMKI or CAMKIV inhibits growth of human AML cells in vitro and in vivo. (3) CAMKI and CAMKIV rescue PirB deficiency in a mouse AML model, and deletion of CAMKIV decreased the activity of AML-SCs. (4) CAMKIV supports the development of acute lymphoblastic leukemia in a mouse model (unpublished data). These results are in agreement with the previous reports that CAMKIV is 
required for HSC activity and CAMKII suppresses differentiation and stimulates proliferation of myeloid leukemia cells [10]. Because we used the MLL-AF9 fusion oncogene to initiate AML in the mouse model, our results suggest that CAMKs play a critical role in the maintenance and progression of AML.

Because CAMKs network with many different signaling pathways, and the signaling of ITAM or ITIM receptors is rather divergent [23], it is possible that CAMKs only represent a critical branch of the total signaling cascades linked to LILRB2/PirB. In general SHP-1 and SHP-2 act immediately downstream of an ITIM-containing inhibitory receptor such as LILRB2/ PirB to initiate further signaling to regulate cell fates. While we showed that SHP-1/2 can induce CAMK activation in AML cells $[1,11]$, it will be important to determine whether other signaling molecules act downstream of LILRB/SHP in leukemia cells. In addition, although we can use serial transplantation to measure AML-SC function and self-renewal, because AML-SCs were not purified to homogeneity in our study, it remains to be determined whether this signaling network differs in stem cells and in more differentiated AML cells.

\section{Conclusion}

We demonstrated that CAMKs are important signaling molecules to support self-renewal and survival in AML cells. Our results thus revealed one mechanism by which the ITIM-containing inhibitory receptors support stem cell activity and AML development and also have important implications in development of new therapeutic strategies in leukemia treatment. It is known that deletion of PirB from normal hematopoiesis or adult HSCs does not have significant in vivo effects [1], and Camk4-knockout mice had only minor defects in HSCs [10]. By contrast, the loss of function of LILRB2/PirB or CAMKs are detrimental to AML development. It is therefore likely that inhibition of CAMKs will be effective in treating leukemia with minimal side effects in the hematopoietic system.

\section{Additional file}

Additional file 1: Figure S1. Western blot showed expression level of CAMKI and CAMKIV in each samples of Fig. 1d. Figure S2. CaMKIV is highly expressed in AML-SC enriched population. (a-b) The expression of PirB (a) and CaMKIV (b) in different normal BM populations as determined by realtime RT-PCR $(n=3)$. (c) CaMKIV expression in total, YFP ${ }^{+} M a c-1{ }^{+} K i t^{-}$, and YFP ${ }^{+}$Mac- $-{ }^{+} \mathrm{Kit}^{+}$BM AML cells as determined by real-time RT-PCR $(n=3) .{ }^{*} p<0.05$. Error bars, s.e.m. Figure S3. Phosphorylation of CAMKIV is greater in myeloid cell lines (U937 and MV4-11) than in ALL cell lines (KASUMI-2 and SUP-B15).

Figure S4. MV4-11 cell growth was inhibited by three different gRNAs targeting camk1. Cell numbers were calculated four days post doxycycline (DOX $1 \mu \mathrm{g} / \mathrm{ml})$ inducement (a). Western blot showed silent effect of gRNAs targeting CAMK1 (b). (PDF $660 \mathrm{~kb})$

\section{Abbreviations}

AML: Acute myeloid leukemia; Angptls: Angiopoietin-like proteins;

CAMK: $\mathrm{Ca}(2+) /$ calmodulin-dependent protein kinases; ITIM: Immunoreceptor tyrosine-based switch motif; LILRB2: Leukocyte immunoglobulin-like receptor B2; PirB: Ortholog-paired Ig-like receptor; WT: Wild-type

\section{Acknowledgements}

Not applicable

\section{Funding}

This work was supported by NIH (1R01CA172268), the Leukemia \& Lymphoma Society (1024-14 and TRP-6024-14), March of Dimes Foundation (1-FY14-201), the Cancer Prevention and Research Institute of Texas (RP140402, DP150056), Robert A. Welch Foundation Award (I-1834), and Fundamental Research Funds for the Central Universities of China (No. 5006-851008).

\section{Availability of data and materials \\ Not applicable}

\section{Authors' contributions}

$X K, C C$, and $C C Z$ contributed to the experimental design, performed the experiments, interpreted the data, and contributed to the writing of the manuscript. CW, MZ, GW, HC, XC, and LW contributed to the experimental performance and interpretation. ZL, HG, ZC, MM, JH, and HW contributed to the in silicon data analysis and interpretation. All authors read and approved the final manuscript.

\section{Ethics approval and consent to participate}

Not applicable

\section{Consent for publication}

Not applicable

\section{Competing interests}

The authors declare that they have no competing interests.

\section{Publisher's Note}

Springer Nature remains neutral with regard to jurisdictional claims in published maps and institutional affiliations.

\section{Author details}

'Departments of Physiology and Developmental Biology, University of Texas Southwestern Medical Center, 5323 Harry Hines Boulevard, Dallas, TX 75390, USA. ${ }^{2}$ Center for Precision Medicine, Department of Medicine, University of Missouri, 1 Hospital Drive, Columbia, MO 65212, USA. ${ }^{3}$ Department of Hematology, The First Affiliated Hospital of Guangzhou Medical University, Guangzhou, China. ${ }^{4}$ School of Life Science and Medicine, Dalian University of Technology, Liaoning 124221, China. ${ }^{5}$ Department of Electrical and Computer Engineering, Missouri University of Science and Technology, Rolla, MO 65409, USA. ${ }^{6}$ Department of Laboratory Medicine, University of California San Francisco, San Francisco, CA 94143, USA. ${ }^{7}$ Department of Systems Biology, Beckman Research Institute, Monrovia, CA 91016, USA. ${ }^{8}$ Department of Pathology, University of California San Diego, La Jolla, CA 92093, USA.

Received: 17 August 2017 Accepted: 12 February 2018 Published online: 27 February 2018

\section{References}

1. Zheng J, Umikawa M, Cui $C$, et al. Inhibitory receptors bind ANGPTLS and support blood stem cells and leukaemia development. Nature. 2012:485:656-60.

2. Deng M, Lu Z, Zheng J, et al. A motif in LILRB2 critical for Angpt12 binding and activation. Blood. 2014:124:924-35.

3. $\mathrm{Ma} \mathrm{G}$, Pan PY, Eisenstein $\mathrm{S}$, et al. Paired immunoglobin-like receptor-B regulates the suppressive function and fate of myeloid-derived suppressor cells. Immunity. 2011:34:385-95.

4. Takai T, Nakamura A, Endo S. Role of PIR-B in autoimmune glomerulonephritis. J Biomed Biotechnol. 2011;2011:275302.

5. Blery M, Kubagawa H, Chen CC, et al. The paired Ig-like receptor PIR-B is an inhibitory receptor that recruits the protein-tyrosine phosphatase SHP-1. Proc Natl Acad Sci U S A. 1998;95:2446-51. 
6. Maeda A, Kurosaki M, Ono M, et al. Requirement of $\mathrm{SH2}$-containing protein tyrosine phosphatases SHP-1 and SHP-2 for paired immunoglobulin-like receptor B (PIR-B)-mediated inhibitory signal. J Exp Med. 1998;187:1355-60.

7. Uehara T, Blery M, Kang DW, et al. Inhibition of lgE-mediated mast cell activation by the paired Ig-like receptor PIR-B. J Clin Invest. 2001;108:1041-50.

8. Chan RJ, Feng GS. PTPN11 is the first identified proto-oncogene that encodes a tyrosine phosphatase. Blood. 2007;109:862-7.

9. Si J, Collins SJ. Activated Ca2+/calmodulin-dependent protein kinase Ilgamma is a critical regulator of myeloid leukemia cell proliferation. Cancer Res. 2008;68:3733-42.

10. Kitsos CM, Sankar U, Illario M, et al. Calmodulin-dependent protein kinase IV regulates hematopoietic stem cell maintenance. J Biol Chem. 2005;280:33101-8

11. Kang X, Lu Z, Cui C, et al. The ITIM-containing receptor LAIR1 is essential for acute myeloid leukaemia development. Nat Cell Biol. 2015;17:665-77.

12. Cheng JC, Kinjo K, Judelson DR, et al. CREB is a critical regulator of normal hematopoiesis and leukemogenesis. Blood. 2008;111:1182-92.

13. Esparza SD, Chang J, Shankar DB, et al. CREB regulates Meis1 expression in normal and malignant hematopoietic cells. Leukemia. 2008;22:665-7.

14. Syken J, Grandpre T, Kanold PO, et al. PirB restricts ocular-dominance plasticity in visual cortex. Science. 2006;313:1795-800.

15. Chen $X$, Zheng J, Zou Y, et al. IGF binding protein 2 is a cell-autonomous factor supporting survival and migration of acute leukemia cells. J Hematol Oncol. 2013;6:72.

16. Xie J, Chen X, Zheng J, et al. IGF-IR determines the fates of BCR/ABL leukemia. J Hematol Oncol. 2015;8:3.

17. Wang T, Wei JJ, Sabatini DM, et al. Genetic screens in human cells using the CRISPR-Cas9 system. Science. 2014;343:80-4.

18. Zheng J, Umikawa M, Zhang S, et al. Ex vivo expanded hematopoietic stem cells overcome the MHC barrier in allogeneic transplantation. Cell Stem Cell. 2011;9:119-30

19. Krivtsov AV, Twomey D, Feng Z, et al. Transformation from committed progenitor to leukaemia stem cell initiated by MLL-AF9. Nature. 2006;442:818-22.

20. Somervaille TC, Cleary ML. Identification and characterization of leukemia stem cells in murine MLL-AF9 acute myeloid leukemia. Cancer Cell. 2006;10:257-68

21. Lu Z, Xie J, Wu G, et al. Fasting selectively blocks development of acute lymphoblastic leukemia via leptin-receptor upregulation. Nat Med. 2017;23:79-90.

22. Sun P, Enslen $H$, Myung PS, et al. Differential activation of CREB by Ca2 +/calmodulin-dependent protein kinases type II and type IV involves phosphorylation of a site that negatively regulates activity. Genes Dev. 1994:8:2527-39.

23. Ivashkiv LB. Cross-regulation of signaling by ITAM-associated receptors. Nat Immunol. 2009:10:340-7.

24. Lukk M, Kapushesky M, Nikkila J, et al. A global map of human gene expression. Nat Biotechnol. 2010;28:322-4.

\section{Submit your next manuscript to BioMed Central and we will help you at every step:}

- We accept pre-submission inquiries

- Our selector tool helps you to find the most relevant journal

- We provide round the clock customer support

- Convenient online submission

- Thorough peer review

- Inclusion in PubMed and all major indexing services

- Maximum visibility for your research

Submit your manuscript at www.biomedcentral.com/submit
Biomed Central 